Voix et Images

\title{
La littérature selon Saint-Sulpice et Gérard Tougas
}

\section{Jacques Michon}

Volume 8, numéro 3, printemps 1983

Jacques Ferron

URI : https://id.erudit.org/iderudit/200407ar

DOI : https://doi.org/10.7202/200407ar

Aller au sommaire du numéro

Éditeur(s)

Université du Québec à Montréal

ISSN

0318-9201 (imprimé)

1705-933X (numérique)

Découvrir la revue

Citer ce compte rendu

Michon, J. (1983). Compte rendu de [La littérature selon Saint-Sulpice et Gérard

Tougas]. Voix et Images, 8(3), 511-513. https://doi.org/10.7202/200407ar d'utilisation que vous pouvez consulter en ligne.

https://apropos.erudit.org/fr/usagers/politique-dutilisation/ 


\title{
La littérature selon Saint-Sulpice et Gérard Tougas
}

\author{
par Jacques Michon, Université de Sherbrooke
}

LAJEUNESSE, Marcel, les Sulpiciens et la Vie culturelle à Montréal au XIXe siècle, Montréal, Fides, 1982, 278 p.

Voici une étude qui concerne les conditions de possibilité de notre littérature au XIXe siècle. Vers 1840, après les querelles politiques et institutionnelles des années 1830, les élites cañadiennes.françaises découvrent l'urgence de créer un "système cohérent d'instruction publique". Les Sulpiciens conscients de la nécessité de l'instruction mais aussi de ses dangers, entreprennent d'orienter ce mouvement dans la bonne direction. Pour annuler l'influence des mauvais livres, ils fondent "I'Oeuvre des bons livres" (1844-1856), une sorte de bibliothèque paroissial qui deviendra plus tard le "Cabinet de lecture paroissial" (1857-1867) qui comprend une bibliothèque, un cercle littéraire où l'on discute et donne des conférences, et un périodique, l'Écho du cabinet de lecture paroissia/ (1859-1875). Durant les années 1850 et 60 le Cabinet représentera l'antidote, le vaccin ultramontain contre l'influence libérale de l'Institut canadien.

Marcel Lajeunesse fait donc l'histoire de ces institutions sulpiciennes qui seront a l'origine du Cercle Ville-Marie (1884-1910) et surtout de la bibliothèque Saint-Sulpice (1910). L'auteur nous donne de précieux renseignèments sur le catalogue de la bibliotheque, son contenu, son évolution, la circulation des volumes et sur le statut social de ses lecteurs et de ses membres qui se recrutaient surtout; semble-t-il, parmi les clercs et la petite bourgeoisie bien pensante. II nous donne également un aperçu du contenu des conférences prononcées au Cercle entre 1857 et 1867 . On apprend, entre autres, que $25,5 \%$ des sujets traités avaient trait aux arts littérature, musique, beauxarts). Les auditeurs étaient prévenus contre "l'influence funeste des mauvais romans", contre Victor Hugo et encouragés à lire la littérature nationale et chrétienne.

Avec la création du Cercle Ville-Marie à la fin du siècle qui regroupe surtout des étudiants de l'Université Laval à Montréal fondée en 1876, la bibliothèque évolue, "elle n'était plus une bibliothèque de 'bons livres' au sens strict. Elle devenait une bibliotheque générale de lecture et de consultation" (p. 196). On voit apparaitre dans ses rangs les ainés et les pères des futurs membres de l'Ecole littéraire de Montréal, comme 
Gonzalve Desaulniers et P.-A.-T. de Montigny. En 1898 les Sulpiciens fondent une chaire de littérature française qu'ils confient à un professeur de Paris. On y voit passer Ferdinand Brunetière et René Doumic. Inutile de dire que ce n'est pas en cette compagnie que les écrivains québécois qui changeront la littérature vont se ressourcer ou se retrouver. Pour voir certains changements dans la conception de la littérature, il faut regarder du côté de certains périodiques qui osent braver l'Index, comme Canada Revue et l'Écho des jeunes qui publient des textes de Zola et Verlaine, du côté du Cercle Saint-Henri et de la bibliothèque de l'Institut Fraser (qui avait hérité du fonds de l'Institut canadien) où Albert Laberge découvrira les contes de Maupassant vers 1890.

Bien que les entreprises sulpiciennes aient été mises sur pied au départ pour résister au progrès des idées nouvelles, on constate que, à terme, cette stratégie s'est lentement effritée pour donner lieu a l'une des premières bibliothèques publiques du Québec. C'est dans cette perspective historique que l'auteur situe son travail ("Cette étude rejoint le courant de l'histoire des bibliothèques..." qui constitue une monogra phie importante, bien documentée, minutieuse, qui couvre plus d'un demi-siecle et nous donne de précieux matériaux concernant l'une des rares institutions culturelles de notre XIXe siècle.

TOUGAS, Gérard, Destin littéraire du Québec, Montréal, Québec/Amérique, 1982, 208 p.

S'appuyant sur sa connaissance des littératures américaines et francophones, Gérard Tougas fait de la prospective et prédit le plus brillant avenir à la littérature du Québec. Qui osera le contredire? II constate d'abord avec une certaine nostalgie que "la France, pays vieillissant, qui n'arrive pas à surmonter les divisions cancéreuses de son passé", n'est plus la puissance littéraire qu'elle a été et que les littératures francophones hors de France satelliş̌es par Paris, n'ont pas les moyens de se distinguer et d'affirmer leur autonomie. Dans ce contexte, grace en partie a la faiblesse de ses consoeurs, la littérature québécoise pourrait donc prétendre au titre de "deuxième littérature de langue française".

Plus positivement $\mathrm{G}$. Tougas va chercher une confirmation de sa thèse du côté des États-Unis dont l'évolution littéraire lui sert de modèle pour tracer l'avenir de notre littérature, qui se trouverait

aujourd'hui au même stade décisif de son développement qu'a connu, au début du siècle, la littérature américaine, quand il s'est agi, dans la conscience collective des écrivains et des érudits, de doter une littérature de sa mythologie et de ses moyens d'action (p. 65).

Ainsi apparaît sous sa plume l'idée de destin littéraire. Les littératures de souche européenne suivraient en Amérique une évolution parallèle qui ne serait pas sans rappeler celle des êtres vivants:

Toutes les grandes aires culturelles issues de l'ancienne domination européenne ont progressé selon les lois de la nature. L'enfant, pendant ses années d'apprentissage, cherche instinctivement la protection du père. Devenu grand, il s'émancipe. (p. 21)

Ayant atteint l'âge adulte, le Québec se détacherait ainsi de la mère patrie. 
Si l'auteur n'hésite pas à recourir aux simplifications anthropomorphiques de ce genre pour appuyer son propos, il devient plus convaincant lorsqu'il s'arrète à des démonstrations plus précises et spécifiques au champ littéraire; lorsqu'il relate, entre autres, l'évolution récente de l'édition, de l'enseignement, de la recherche et de la critique au Québec, et lorsqu'il s'emploie à montrer l'effort des écrivains actuels pour "se hausser a une langue littéraire nationale". La recherche d'un équilibre entre les niveaux de langue, la recherche d'une langue mitoyenne entre le style soutenu et la langue populaire, placerait l'écrivain québécois dans une position semblable à celle de l'écrivain américain de l'entre-deux-guerres qui a fini, après plusieurs tergiversations, par se forger un idiome propre.

Ces aperçus, sans doute les plus intéressants du livre, gagneraient cependant a être moins marqués par les partis pris esthétiques et idéologiques de l'auteur. Ainsi les propos sur la prose de G. Bessette et de G. LaRoque sont un peu trop taillés sur mesure et d'autant plus suspects qu'ils paraissent dans une collection justement dirigée par ce dernier. En outre, la recherche d'un nouvel équilibre linguistique au Québec n'a la faveur de $M$. Tougas que dans la mesure où elle représente un frein, une résistance au nivellement par le bas dont seraient victimes les grandes cultures. La France et l'Angleterre, sous la pression de la culture américaine, seraient vouées à une dégradation sans rémission, alors que le Québec, lui, représenterait, momentanément du moins, la résistance à la barbarie collectiviste de l'homme moyen et de la langue populaire généralisée:

Il existe donc un frein à l'accréditation de la langue populaire. Pourtant, si notre analyse du collectivisme qui est en marche dans le monde est juste, il suffira, pour que les dernières barrières devant la langue du peuple soient emportées comme elles l'ont été aux États-Unis, que la classe moyenne québécoise s'étoffe et s'enrichisse davantage, s'étendant à des couches de plus en plus modestes de la société. (p. 131)

Ces prises de position élitistes sont peut-être goûtées par les "happy few", "ceux qui n'admettent que les nourritures d'exception", à qui G. Tougas destine son ouvrage; pour les autres, elles ne feront que rendre détestables ces propos fondés sur une sociologie trop simpliste.

II est regrettable que la démarche comparatiste adoptée par l'auteur, intéressante en elle-méme, entre autres pour un lecteur soucieux de mesurer la dimension internationale de la littérature québécoise, soit gâtée par cette conception hautaine et réactionnaire de la langue et de la littérature. 